\title{
THE DECAY OF SUBHARMONIC FUNCTIONS OF FINITE ORDER ALONG A RAY
}

\author{
J. M. ANDERSON AND A. M. ULANOVSKY
}

(Communicated by Albert Baernstein II)

\begin{abstract}
A result is proved relating the growth of a subharmonic function $u(z)$ of finite lower order at least one, along a ray, to the quantity

$$
B(r)=\sup \{u(z):|z|<r\} .
$$

This sharpens a previous result of the second author when the lower order is finite. An example is constructed to show that the result obtained is best possible.
\end{abstract}

\section{INTRODUCTION}

Let $u(z)$ be a subharmonic function (s.f.) in $U$ where $U$ denotes either the complex plane $\mathbf{C}$ or the sector $U(\theta)=\{z:|\arg z|<\theta\}$ where $0<\theta \leq \pi$. If

$$
B(r)=\sup _{|z|<r, z \in U} u(z),
$$

then the lower order $\lambda$ of $u(z)$ is defined by

$$
\lambda=\liminf _{r \rightarrow \infty} \frac{\log B(r)}{\log r} .
$$

It is well known that a s.f. $u$ cannot decay too fast along a ray in comparison with $B(r)$. For convenience we consider the positive ray.

Theorem A. (a) If $0 \leq \lambda<1$, then

$$
u(r) \geq(\cos \pi \lambda) B(r)(1+o(1)),
$$

for a sequence $r=r_{k} \rightarrow \infty$.

(b) If $\lambda \geq 1$, then

$$
u(r) \geq-B(r)(1+o(1)),
$$

for a sequence $r=r_{k} \rightarrow \infty$.

Part (a) is the $\cos \pi \lambda$-theorem, valid also if $u(r)$ is replaced by

$$
A(r)=\inf \{u(z):|z|=r, z \in \mathbf{C}\} .
$$

Received by the editors February 8, 1994 and, in revised form, May 19, 1994.

1991 Mathematics Subject Classification. Primary 30D20, 31A05.

This research has been supported by a British Royal Society Fellowship. The second author gratefully acknowledges the hospitality of the School of Mathematical Sciences at Queen Mary and Westfield College. 
Part (b) is due to A. Beurling ([1]) but, as shown by Hayman [2] and Fryntov (to appear in Proc. Amer. Math. Soc.), the corresponding result for $A(r)$ is false for every $\lambda>1$. This is also discussed in Chapter 6 of [2]. The following result is in [4]:

Theorem B. (a) If $\lambda>1$, then

$$
u(r) \geq-B(r)-\frac{\pi^{2}}{2} \frac{B(r)}{\log B(r)}(1+o(1)),
$$

for a sequence $r=r_{k} \rightarrow \infty$.

(b) If $\lambda=1$, then either $u(x+i y)=a+b x \quad(b<0)$ or

$$
\limsup _{r \rightarrow \infty}(u(r)+B(r))=\infty .
$$

The constant $\pi^{2} / 2$ in the inequality (1) is sharp, as shown in [4]:

Theorem C. Let $\phi(z)=\sum_{k=0}^{\infty} a_{k} z^{k}$, where $a_{k} \geq 0$ and $\sum_{k=1}^{\infty} a_{k}>0$. Then if $u(z)=-\mathfrak{R} \exp \phi(z)$, we have

$$
u(r) \leq-B(r)-\frac{\pi^{2}}{2} \frac{B(r)}{\log B(r)}(1+o(1)), \quad r \rightarrow \infty .
$$

\section{RESULtS}

The present note starts from the observation that the functions of Theorem $\mathrm{C}$ all have infinite lower order. It turns out that (1) can be improved if $\lambda<\infty$.

Theorem 1. Let $u(z)$ be subharmonic in $U(\theta), 0<\theta \leq \pi$, and continuous on the boundary, and suppose further that $\lambda \geq \pi / \theta$. If

$$
\limsup _{r \rightarrow \infty}(u(r)+B(r))<\infty,
$$

then

$$
\int_{1}^{\infty}(u(r)+B(r)) \frac{\log r}{r^{\lambda+1}} d r>-\infty .
$$

The surprising thing, perhaps, in Theorem 1 is the presence of the $\log r$ factor. But Theorem 1 is sharp, as is shown by

Theorem 2. Given any $\lambda$ with $1<\lambda<\infty$ and any positive increasing function $\psi(r) \rightarrow \infty$ as $r \rightarrow \infty$, there is a function $u(z)$, subharmonic in $\mathbf{C}$ with order $\lambda$, satisfying (2) and such that

$$
\int_{1}^{\infty}(u(r)+B(r)) \frac{\psi(r) \log r}{r^{\lambda+1}} d x=-\infty
$$

Theorem 2 can give an improved version of Theorem 2 of [3].

Corollary 1. For any $\lambda>1$ there exists an entire function $f(z)$ of perfectly regular growth of order $\lambda$ such that

$$
\int_{1}^{\infty}(\log m(r)+\log M(r)) \frac{\psi(r) \log r}{r^{1+\lambda}} d r=-\infty
$$

Here, of course

$$
m(r)=\min \{|f(z)|:|z|=r\}, \quad M(r)=\max \{|f(z)|:|z|=r\}
$$


and $\psi(r)$ is, as before, an arbitrary function tending to $\infty$ as $r \rightarrow \infty$. It will be clear from the proof of Theorem 5 that (3) can be achieved for $\lambda=1$, but the corresponding functions are subharmonic only in $U(\pi)$ and not in $\mathbf{C}$. Drasin has shown recently (private communication) that there exists an entire function of order 1 maximal type for which

$$
\log m(r)+\log M(r) \rightarrow-\infty \quad(r \rightarrow \infty),
$$

thus answering a question posed by Hayman in [3]. Because of the abovementioned difficulty, we are unable to extend Corollary 1 to the case $\lambda=1$ and maximal type.

We do not give the proof of Corollary 1. Although our construction yields only a subharmonic function, the necessary adjustments to obtain an entire function are precisely those of [3], Section 2 .

\section{Proof of Theorem 1}

It is enough to consider the case $\theta=\pi$ and $\lambda=1$ (otherwise we consider $v(z)=u\left(z^{1 / \lambda}\right)$ in $\left.U(\pi)\right)$. By subtracting a suitable constant, if necessary, we may also assume, by (2), that

$$
u(r)+B(r)<0,
$$

for $r>0$. For $0<\varepsilon<\pi / 2$ set, for $0<\phi<\pi-\varepsilon$,

$$
u_{\varepsilon}\left(r e^{i \varphi}\right)=u\left(r e^{i(\pi-\varepsilon-\varphi)}\right)+u\left(r e^{i \varphi}\right)
$$

so that $u_{\varepsilon}$ is subharmonic in the sector $V(\varepsilon)=\{z: 0<\arg z<\pi-\varepsilon\}$ and continuous on the boundary. Moreover, from (4)

$$
u_{\varepsilon}(r)<0, \quad u_{\varepsilon}\left(r e^{i(\pi-\varepsilon)}\right)<0,
$$

for all $r>0$. Since $u_{\varepsilon}(z) \leq 2 B(|z|)$, the lower order of $u_{\varepsilon}$ is at most $\lambda$ (which we have taken equal to 1 ). So, the Phragmen-Lindelöf Principle yields that $u_{\varepsilon}\left(r e^{i \varphi}\right)<0$, in $V_{\varepsilon}$. In particular

$$
0>u_{\varepsilon}\left(r e^{i \frac{\pi-\varepsilon}{2}}\right)=2 u\left(r e^{i \frac{\pi-\varepsilon}{2}}\right) .
$$

Since these inequalities hold for every $0<\varepsilon<\pi / 2$ and every $0<\varphi<\pi-\varepsilon$, we conclude that $u(z)<0,0<\arg z<\pi / 2$; and that $\left.u_{0}\left(r e^{i \varphi}\right)\right)=u\left(r e^{i(\pi-\varphi)}\right)+$ $u\left(r e^{i \varphi}\right)<0,0 \leq \varphi \leq \pi / 2$. Similar inequalities hold for $-\pi / 2<\arg z<0$ for $u(z)$ and for $\pi / 2<\varphi<\pi$ for $u_{0}\left(r e^{i \varphi}\right)$. Thus

$$
u(z)<0, \quad y=\mathfrak{I} z>0 ; \quad u_{0}(z)<0, \quad x=\mathfrak{R} z>0 .
$$

From the limiting case $R \rightarrow \infty$ of [2], Lemma 6.1, p. 296, the boundary values $u_{0}(x)$ and $u(i y)$ satisfy

$$
-\infty<\int_{-\infty}^{\infty} \frac{u(i y)}{1+y^{2}} d y<\int_{0}^{\infty} \frac{u(i y)}{1+y^{2}} d y .
$$

Analogously, since $u_{0}(z)<0$ for $\mathfrak{R} z>0$, we have

$$
-\infty<\int_{-\infty}^{\infty} \frac{u_{0}(x)}{1+x^{2}} d x
$$


Moreover, from [2], Lemma 6.6, p. 317,

$$
2 u(i y)=u_{0}(i y) \leq \frac{y}{\pi} \int_{-\infty}^{\infty} \frac{u_{0}(x)}{x^{2}+y^{2}} d x=\frac{4 y}{\pi} \int_{0}^{\infty} \frac{u(x)+u(-x)}{x^{2}+y^{2}} d x
$$

Combining these we obtain, since $u(i y) \leq 0$, that

$$
\begin{aligned}
-\infty & <\int_{0}^{\infty} \frac{u(i y)}{1+y^{2}} d y \leq \int_{1}^{\infty} \frac{u(i y)}{2 y^{2}} d y \\
& \leq \frac{2}{\pi} \int_{0}^{\infty}\left(\int_{e^{-1}}^{\infty} \frac{1}{y\left(x^{2}+y^{2}\right)} d y\right)(u(x)+u(-x)) d x \\
& \leq \frac{1}{\pi} \int_{e^{-1}}^{\infty} \frac{1+\log x}{x^{2}+1}(u(x)+u(-x)) d x,
\end{aligned}
$$

which proves Theorem 1.

\section{Proof OF TheOREM 2}

We may suppose that $1<\lambda \leq 2$. For if $\lambda>2$ we may write $\lambda=n \lambda_{0}$ where $n \in \mathbf{N}$ and $1<\lambda_{0} \leq 2$. If $u_{0}(z)$ is the subharmonic function whose existence is asserted for $\lambda_{0}$ and for which the integral (3) diverges, then $u(z)=u_{0}\left(z^{n}\right)$ satisfies (3) for a given value $\lambda$. The function $u(z)$ will have the form, for $|\phi| \leq \pi$

$$
u\left(r e^{i \phi}\right)=-\mathfrak{R} \int_{0}^{\lambda-1} z^{\lambda-t} p(t) d t, \quad z=r e^{i \phi},
$$

for a suitable function $p(t)$.

For the given function $\psi(t)$ of Theorem 2 we set

$$
\begin{aligned}
& \psi_{1}(t)=\int_{0}^{\infty} \psi\left(e^{r / t}\right) r e^{-r} d r \\
& \psi_{2}(t)=\int_{0}^{\infty} \psi\left(e^{r / t}\right) e^{-t} d r
\end{aligned}
$$

Since $\psi(r) \rightarrow \infty$ as $r \rightarrow \infty$, we conclude that

$$
\begin{gathered}
\psi_{j}(t) \rightarrow \infty, \quad t \rightarrow 0, j=1,2 ; \\
\psi_{1}(t)-4 \psi_{2}(t) \rightarrow \infty, \quad t \rightarrow 0 .
\end{gathered}
$$

We now choose the function $p(t)$ to satisfy the following conditions:

$$
p(0)=0 ; \quad p^{\prime}(t)>0, \quad 0<t<\lambda-1 ; \quad \int_{0}^{\lambda-1} p(t) d t=1 ;
$$

and

$$
\int_{0}^{\lambda-1} p(t)\left(\psi_{1}(t)-4 \psi_{2}(t)\right) d t=\infty
$$

Clearly such a function $p(t)$ exists. Now $u(z)$ defined by (5) is subharmonic in $U(\pi)$. Moreover, since $\sin \pi(\lambda-t)<0$ for $0<t<\lambda-1$, we have $(\partial u / \partial \varphi)(-r)<0$, for all $r>0$. Using this inequality it is easy to establish that, for sufficiently small $\rho$,

$$
u(-r) \leq \frac{1}{2 \pi} \int_{0}^{2 \pi} u\left(-r+\rho e^{i \theta}\right) d \theta
$$


Thus $u(z)$ is, in fact, subharmonic in $\mathbf{C}$. We remark that it is precisely here that our argument is unable to deal with the case $\lambda=1$.

We define $\alpha(r)$, perhaps not uniquely, by the equation

$$
B(r)=u\left(r e^{i \alpha(r)}\right), \quad r>0,
$$

and note that

$$
(\partial u / \partial \varphi)\left(r e^{i \alpha(r)}\right)=\int_{0}^{\lambda-1} r^{\lambda-t}(\lambda-t) p(t) \sin (\alpha(r)(\lambda-t)) d t=0 .
$$

Simple arguments prove that $\alpha(r)>\pi / \lambda$ and that $\alpha(r) \rightarrow \pi / \lambda, r \rightarrow \infty$.

We set $\varepsilon(r)=\lambda \alpha(r)-\pi$ so that

$$
\begin{aligned}
u(r)+B(r) & =-2 \int_{0}^{\lambda-1} r^{\lambda-t} \cos ^{2}\left((\lambda-t) \frac{\pi+\varepsilon(r)}{2 \lambda}\right) p(t) d t \\
& =-2 \int_{0}^{\lambda-1} r^{\lambda-t} \sin ^{2}\left(\frac{\varepsilon(r)}{2}-\frac{t(\pi+\varepsilon(r))}{2 \lambda}\right) p(t) d t
\end{aligned}
$$

Using the estimates $\sin x>x / 2$ for $|x|<\pi / 6$ and $|\varepsilon(r) / 2-t(\pi+\varepsilon(r)) / 2 \lambda|<$ $\pi / 6$ for $0<t<\lambda / 4$ as $r \rightarrow \infty$, we obtain, as $r \rightarrow \infty$,

$$
\begin{aligned}
u(r)+B(r) & \leq-\frac{1}{2} \int_{0}^{\lambda-1} r^{\lambda-t}\left(\frac{\varepsilon(r)}{2}-t \frac{\pi+\varepsilon(r)}{2 \lambda}\right)^{2} p(t) d t+O\left(r^{\frac{3 \lambda}{4}}\right) \\
& =-\frac{(\pi+\varepsilon(r))^{2}}{8 \lambda^{2}} \int_{0}^{\lambda-1} r^{\lambda-t}(t-\delta(r))^{2} d t+O\left(r^{\frac{3 \lambda}{4}}\right) \\
& \leq-\frac{\pi^{2}}{16 \lambda^{2}} \int_{0}^{\lambda-1} r^{\lambda-t}(t-\delta(r))^{2} p(t) d t
\end{aligned}
$$

where, for convenience, $\delta(r)=\lambda \varepsilon(r) /(\pi+\varepsilon(r))$.

Inspection shows that the last integral attains its minimum when $\delta(r)=$ $\delta_{0}(r)$, where

$$
\delta_{0}(r)=\frac{\int_{0}^{\lambda-1} t r^{-t} p(t) d t}{\int_{0}^{\lambda-1} r^{-t} p(t) d t}
$$

Now

$$
\int_{0}^{\lambda-1} t r^{-t} p(t) d t=-\left.\frac{t p(t) r^{-t}}{\log r}\right|_{0} ^{\lambda-1}+\frac{1}{\log r} \int_{0}^{\lambda-1} r^{-t}\left(p(t)+t p^{\prime}(t)\right) d t
$$

and since $p^{\prime}(t)>0$, it follows that $\delta_{0}(r)<2 / \log r$ as $r \rightarrow \infty$. We conclude that

$$
u(r)+B(r) \leq-\frac{\pi^{2}}{16 \lambda^{2}} \int_{0}^{\lambda-1} r^{\lambda-t}\left(t^{2}-\frac{4 t}{\log r}\right) p(t) d t
$$


The proof now follows immediately. From (6) we obtain

$$
\begin{gathered}
\int_{1}^{\infty}(u(r)+B(r)) \frac{\psi(r) \log r}{r^{\lambda+1}} d r \\
\leq \frac{\pi^{2}}{8 \lambda^{2}}\left(-\int_{0}^{\infty} \int_{0}^{\lambda-1} \psi\left(e^{r}\right) r e^{-t r} t^{2} p(t) d t d r\right. \\
\left.\quad+4 \int_{0}^{\infty} \int_{0}^{\lambda-1} \psi\left(e^{r}\right) e^{-t r} t p(t) d t d r\right) \\
=-\frac{\pi^{2}}{8 \lambda^{2}} \int_{0}^{\lambda-1} p(t)\left(\psi_{1}(t)-4 \psi_{2}(t)\right) d t=-\infty .
\end{gathered}
$$

Theorem 2 is proved.

\section{REFERENCES}

1. A. Beurling, Some theorems on boundedness of analytic functions, Duke Math. J. 16 (1949), 355-359.

2. W. K. Hayman, Subharmonic functions, Vol. 2, Academic Press, New York, 1989.

3. 171-212.

4. A. M. Ulanovsky, How fast can a subharmonic function decay along a ray? (to appear).

Department of Mathematics, University College London, London WC1E 6BT, United KINGDOM

Institute for Low Temperature, Physics, 47, Lenin Avenue 310164, Kharkov, Ukraine

E-mail address: ulanovskii math25.ilt.kharkov.ua 